\title{
Tubular Anisotropy for 2D Vessel Segmentation
}

\author{
Fethallah Benmansour, Laurent D. Cohen \\ CEREMADE, UMR CNRS 7534, Université Paris Dauphine, \\ Place du Maréchal De Lattre De Tassigny, \\ 75775 PARIS CEDEX 16 - FRANCE \\ benmansour, cohen@ceremade.dauphine.fr \\ Max W.K. Law and Albert C.S. Chung \\ Lo Kwee-Seong Medical Image Analysis Laboratory, \\ Department of Computer Science and Engineering, \\ The Hong Kong University of Science and Technology, Hong Kong \\ maxlawwk, achung@cse.ust.hk
}

\begin{abstract}
In this paper, we present a new approach for segmentation of tubular structures in $2 D$ images providing minimal interaction. The main objective is to extract centerlines and boundaries of the vessels at the same time. The first step is to represent the trajectory of the vessel not as a $2 D$ curve but to go up a dimension and represent the entire vessel as a $3 D$ curve, where each point represents a $2 D$ disc (two coordinates for the center point and one for the radius). The $2 D$ vessel structure is then obtained as the envelope of the family of discs traversed along this $3 D$ curve. Since this $2 D$ shape is defined simply from a $3 D$ curve, we are able to fully exploit minimal path techniques to obtain globally minimizing trajectories between two or more user supplied points using front propagation. The main contribution of our approach consists on building a multi-resolution metric that guides the propagation in this $3 D$ space. We have chosen to exploit the tubular structure of the vessels one wants to extract to built an anisotropic metric giving higher speed on the center of the vessels and also when the minimal path tangent is coherent with the vessel's direction. This measure is required to be robust against the disturbance introduced by noise or adjacent structures with intensity similar to the target vessel. Indeed, if we examine the flux of the projected image gradient along a given direction on a circle of a given radius (or scale), one can prove that this flux is maximal at the center of the vessel, in its direction and with its exact radius. This approach is called optimally oriented flux. Combining anisotropic minimal paths techniques and optimally oriented flux we obtain promising results on noisy synthetic and real data.
\end{abstract}

\section{Introduction}

In this paper we deal with the problem of finding a complete segmentation of tubular structures like vessels. The main objective is to extract at the same time the centerline of the tubular structure and its boundary. During the last two decades, the extraction of vascular objects such as the blood vessel, coronary arteries, or other tube-like structures has attracted the attention of more and more researchers. Various methods such as vascular image enhancement methods $[15,8,14,6]$, or others were proposed, see [5, 13] for a complete survey. Some of these methods extract the vessel boundary directly, and then use thinning methods to find its centerline. Other methods extract only the centerline and then estimate the vessel width to extract its boundary. Deschamps and Cohen [3] proposed to use the minimal path method to find the centerline as well as a rough estimate of the vessel. The minimal path technique introduced by Cohen and Kimmel [2] captures the global minimum curve between two points given by the user. This leads to the global minimum of an active contour energy. Since then, the minimal path method has been improved by many researchers, and adapted to anisotropic media as done by Jbabdi et al for tractography [7]. Unfortunately, despite their numerous advantages, classical minimal path techniques exhibit some disadvantages. First, precise vessel boundary extraction can be very difficult, even in 2D where the vessel's boundary can be completely described by two curves. Second, the path given by the minimal path technique does not always yield to the centerline of the vessel. A readjustment step is required to obtain a central trajectory. Third, the minimal path technique provides only a trajectory and does not give information about the vessel boundary and local width. 
$\mathrm{Li}$ and Yezzi [10] proposed a new variant of the classical, purely spatial, minimal path technique by incorporating an extra non-spatial dimension into the search space. Each point of the 3D path (after adding the extra dimension for the 2D image) consists of two spatial coordinates plus a third coordinate which describes the vessel thickness at that corresponding 2D point. Thus, each 3D point represents a disc in 2D space, and the vessel is obtained by taking the envelope of these spheres as we move along the 3D curve. A crucial step of this method is to build an adequate potential that drives the propagation. Li and Yezzi [10] proposed different isotropic potentials. As they said in the conclusion of their paper, the proposed potentials are very parameter dependent and they hoped to find more appropriate choice of potential. In particular, one can see in their paper, that the potential used does not yield to a correct detection of the radius when it is not constant (see figure 6 in [10]). An other drawback of Li and Yezzi method is that they did not take into account the vessel orientation. Our first contribution is to take into account the vessel orientation by considering an anisotropic metric. This metric has to make the propagation faster along the centerlines and for the adequate radius. Law and Chung [9] proposed a new scalar descriptor called Optimally Oriented Flux (OOF) for the detection of curvilinear structures. But they did not exploit the orientation given by their descriptor. The major advantage of the OOF technique is that it does not consider the regions in the vicinity of target objects, where background noise or adjacent structures with intensity similar to the target vessels are possibly present. Therefore, the disturbance introduced by the closely located nearby structures is avoided. The second contribution of this paper is to build an anisotropic metric based on the OOF descriptor, its scalar function as well as its orientation. That makes the propagation faster along the vessels center line and for exact associated scale. This means that the path location, orientation and scale (radius) have to be coherent with the local geometry of the image extracted by the OOF.

In section 2, we give some background on the minimal path method and Anisotropic Fast Marching. In section 3 the Optimally Oriented Flux descriptor is presented and the metric construction is detailed in section 4. In section 5, results on synthetic and real data are shown. Finally, conclusions and perspectives follow in section 6 .

\section{Background on Minimal Paths Method}

A minimal path, first introduced in the isotropic $(\mathcal{P}$ does not depend on the orientation of the path) case [2], is a pathway minimizing the energy functional,

$$
E(\gamma)=\int_{\gamma} \mathcal{P}\left(\gamma(s), \gamma^{\prime}(s)\right) \mathrm{d} s
$$

where, $\mathcal{P}\left(\gamma(),. \gamma^{\prime}().\right)=\sqrt{\gamma^{\prime}(.)^{T} \mathcal{M}(\gamma(.)) \gamma^{\prime}(.)}$ describes an infinitesimal distance along a pathway $\gamma$ relative to a metric tensor $\mathcal{M}$ (symmetric definite positive). Thus, we are considering only the case of an elliptic medium which is a particular case of the Finsler metric introduced in active contour framework by Melonakos et al. in [12]. In the isotropic case $\mathcal{M}()=.\mathcal{P}^{2}()$.$I , where I$ is the identity matrix. A curve connecting $\mathbf{p}_{1}$ to $\mathbf{p}_{2}$ that globally minimizes the above energy (1) is a minimal path between $\mathbf{p}_{1}$ and $\mathbf{p}_{2}$, noted $\mathcal{C}_{\mathbf{p}_{1}, \mathbf{p}_{2}}$.

The solution of this minimization problem is obtained through the computation of the minimal action map $\mathcal{U}$ : $\Omega \rightarrow \mathbb{R}^{+}$associated to $\mathbf{p}_{1}$ on the domain $\Omega$ which is a $3 \mathrm{D}$ domain in our case since the considered minimal path (after adding the scale dimension) is a 3D path. The minimal action is the minimal energy integrated along a path between $\mathbf{p}_{1}$ and any point $\mathbf{x}$ of the domain $\Omega$ :

$$
\forall \mathbf{x} \in \Omega, \mathcal{U}(\mathbf{x})=\min _{\gamma \in \mathcal{A}_{\mathbf{p}_{1}, \mathbf{x}}}\left\{\int_{\gamma} \mathcal{P}\left(\gamma(s), \gamma^{\prime}(s)\right) \mathrm{d} s\right\},
$$

where $\mathcal{A}_{\mathbf{p}_{1}, \mathbf{x}}$ is the set of paths linking $\mathbf{x}$ to $\mathbf{p}_{1}$. The values of $\mathcal{U}$ may be regarded as the arrival times of a front propagating from the source $\mathbf{p}_{1}$ with oriented velocity related to the metric tensor $\mathcal{M}^{-1}$. $\mathcal{U}$ satisfies the Eikonal equation

$$
\|\nabla \mathcal{U}(\mathbf{x})\|_{\mathcal{M}^{-1}(\mathbf{x})}=1 \text { for } \mathbf{x} \in \Omega \text {, and } \mathcal{U}\left(\mathbf{p}_{1}\right)=0,
$$

where $\|\mathbf{v}\|_{M}=\sqrt{\mathbf{v}^{T} M \mathbf{v}}$. The map $\mathcal{U}$ has only one local minimum, the point $\mathbf{p}_{1}$, and the filed lines of $\mathcal{M}^{-1} \nabla \mathcal{U}$ satisfy the Euler-Lagrange equation of functional (1). Thus, the minimal path $\mathcal{C}_{\mathbf{p}_{1}, \mathbf{p}_{2}}$ can be retrieved with a simple gradient descent on $\mathcal{U}$ from $\mathbf{p}_{2}$ to $\mathbf{p}_{1}$ (see Fig. 1), solving the following ordinary differential equation with standard numerical methods like Heun's or Runge-Kutta's :

$$
\left\{\begin{aligned}
\frac{\mathrm{d} \mathcal{C}_{\mathbf{p}_{1}, \mathbf{p}_{2}}}{\mathrm{~d} s}(s) & \propto-\mathcal{M}^{-1}\left(\mathcal{C}_{\mathbf{p}_{1}, \mathbf{p}_{2}}(s)\right) \nabla \mathcal{U}_{1}\left(\mathcal{C}_{\mathbf{p}_{1}, \mathbf{p}_{2}}(s)\right), \\
\mathcal{C}_{\mathbf{p}_{1}, \mathbf{p}_{2}}(0) & =\mathbf{p}_{2} .
\end{aligned}\right.
$$

Proof of (3) and (4) can be found in [17, 7]. On figure 1 , we show some examples of the minimal path method on an isotropic case and an anisotropic one. On the first image of figure 1 the metric is isotropic and the potential $\mathcal{P}$ in the grey region is half of the one on the white region. Isolevel sets of the minimal action map associated to the source point $\mathbf{p}_{1}$ are displayed and so the minimal path $\mathcal{C}_{\mathbf{p}_{1}, \mathbf{p}_{2}}$. The second image represents a metric $\mathcal{M}$. We took $\mathcal{M}=\left(\begin{array}{cc}1 & 0.3 \\ 0.3 & 1\end{array}\right)$ on the left side image and $\mathcal{M}=\left(\begin{array}{cc}1 & -0.6 \\ -0.6 & 1\end{array}\right)$ on the right side. On the last image, the minimal action map $\mathcal{U}$ associated to the metric $\mathcal{M}$ and to the source point $\mathbf{p}_{1}$ is shown. The minimal path $\mathcal{C}_{\mathbf{p}_{1}, \mathbf{p}_{2}}$ is found by solving equation (4). 


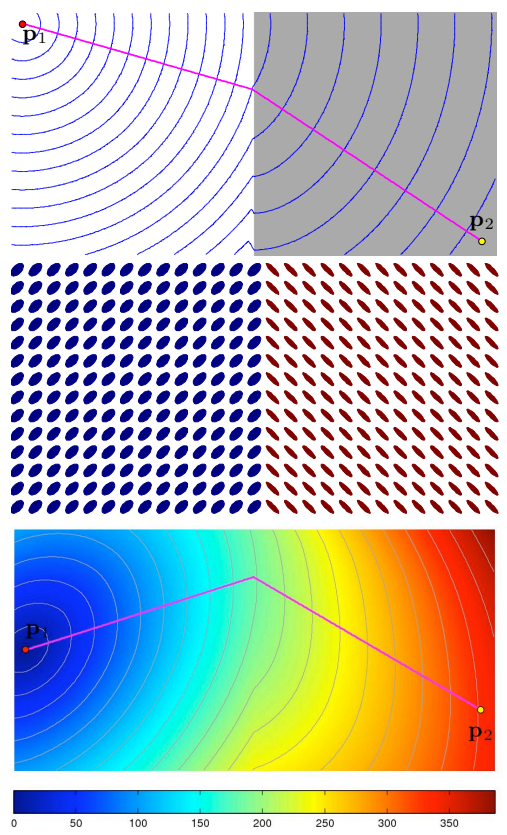

Figure 1. Minimal path examples on an isotropic case on the first image. On the second image, visualization by small ellipses of eigenvalues of a metric constant on each half side of the image. On the last one, the minimal action map associated to the source point $\mathbf{p}_{1}$ with the minimal path $\mathcal{C}_{\mathbf{p}_{1}, \mathbf{p}_{2}}$.

The Fast Marching Method (FMM) is a numerical method introduced by Sethian in [16] and Tsitsiklis in [18] for efficiently solving the isotropic Eikonal equation on a cartesian grid. The central idea behind the FMM is to visit grid points in an order consistent with the way wavefronts of constant action propagate. It leads to a single-pass algorithm for solving equation (3) and computing the minimal action map $\mathcal{U}$. Tsitsiklis's method relies on minimizing directly the energy functional of equation (1) while Sethian's method uses the Eikonal equation. Both methods are suitable for isotropic metric, but they fail for anisotropic metric [1]. To deal with anisotropy, Sethian and Vladimirsky [17] proposed an update scheme that converges to the viscosity solution of the anisotropic Eikonal equation. A simplified scheme, based on the original Tsitsiklis's method [18], was proposed by Lin in [11] to approximate the solution of the anisotropic Eikonal equation. Contrary to Sethian and Vladimirsky's ordered upwind method (OUM) [17], Lin's algorithm does not converge to the viscosity solution of the Eikonal equation. In this paper we used Lin's scheme to solve the anisotropic Eikonal equation, since it is much faster than OUM and the introduced errors do not affect much the extracted geodesics.

The FMM is a front propagation approach that computes the values of $\mathcal{U}$ in increasing order, and the structure of the algorithm is almost identical to Dijkstra's algorithm for computing shortest paths on graphs [4]. In the course of the algorithm, each grid point is tagged as either Alive (point for which $\mathcal{U}$ has been computed and frozen), Trial (point for which $\mathcal{U}$ has been estimated but not frozen) or Far (point for which $\mathcal{U}$ is unknown). The set of Trial points forms an interface between the set of grid points for which $\mathcal{U}$ has been frozen (the Alive points) and the set of other grid points (the Far points). This interface may be regarded as a front expanding from the source until every grid point has been reached. Let us denote by $\mathcal{N}_{\mathrm{M}}(\mathrm{x})$ the set of M neighbors of a grid point $\mathbf{x}$, where $\mathrm{M}=2 \times 3$ since the dimension of $\Omega$ is equal to 3 . Initially, all grid points are tagged as Far, except the source point $\mathbf{p}_{1}$ that is tagged as Trial with estimate value 0 . At each iteration of the FMM one chooses the Trial point with the smallest $\mathcal{U}$ value, denoted by $\mathbf{x}_{\min }$. Then, $\mathbf{x}_{\min }$ is tagged as Alive and the value of $\mathcal{U}$ is updated for each point of the set $\mathcal{N}_{\mathrm{M}}\left(\mathrm{x}_{\min }\right)$ which is either Trial or Far. In order to satisfy a causality condition, the way $\mathcal{U}$ is updated in the vicinity of $\mathbf{x}_{\min }$ requires special care. The iteration ends by tagging every Far point of the set $\mathcal{N}_{\mathrm{M}}\left(\mathbf{x}_{\min }\right)$ as Trial. The algorithm (see Table 1) automatically stops when all grid points are Alive. The key to the speed of the FMM is the use of a priority queue to quickly find the Trial point with the smallest $\mathcal{U}$ value. If Trial points are ordered in a min-heap data structure, the computational complexity of the FMM is $\mathcal{O}(N \log N)$, where $N$ is the total number of grid points.

Table 1 : Fast Marching Method for solving equation (3).

- Notation.

$\mathcal{N}_{\mathrm{M}}(\mathbf{x})$ is the set of $\mathrm{M}$ neighbors of a grid point $\mathbf{x}$,

where $\mathrm{M}=6$ in $3 \mathrm{D}$.

- Initialization.

For each grid point $\mathbf{x}$, do $\operatorname{Set} \mathcal{U}(\mathbf{x}):=+\infty$

Tag $\mathbf{x}$ as Far.

For the source point $\mathbf{p}_{1}$, do

$\operatorname{Set} \mathcal{U}\left(\mathbf{p}_{1}\right):=0$.

Tag $\mathbf{p}_{1}$ as Trial.

- Marching loop.

While the set of Trial points is non-empty, do

Find $\mathbf{x}_{\min }$, the Trial point with the smallest $\mathcal{U}$ value.

Tag $\mathbf{x}_{\min }$ as Alive.

For each point $\mathbf{x}_{\mathrm{m}} \in \mathcal{N}_{\mathrm{M}}\left(\mathbf{x}_{\mathrm{min}}\right)$ which is not Alive, do

$\{u\}:=$ Updatescheme $\left(\mathbf{x}_{\mathrm{m}}, \mathcal{N}_{\mathrm{M}}\left(\mathbf{x}_{\mathrm{m}}\right)\right)$ (see text).

$\operatorname{Set} \mathcal{U}\left(\mathbf{x}_{\mathrm{m}}\right):=u$.

If $\mathbf{x}_{\mathrm{n}}$ is Far, tag $\mathbf{x}_{\mathrm{m}}$ as Trial.

A crucial step of the Fast Marching algorithm is the computation of the weighted distance between the front and the neighbouring voxels in the Trial set. Here, we present a way to estimate this weighted distance in the anisotropic case and only in 3D. Since the distance is anisotropic, we cannot use the standard upwind methods, because they rely on the fact that the geodesics are perpendicular to the level sets of $\mathcal{U}$. To take into account the anisotropy Jbabdi et al 
[7] and Lin [11] considered a set of simplexes that cover the whole neighborhood around a voxel of the narrow band. The choice they made reduces errors on the obtained minimal path but does not guarantee the convergence to the viscosity solution of the Eikonal equation. The definition of a simplex neighboring a point $\mathbf{x}$ is simply a set of three points $\left(\mathrm{x}_{1}, \mathrm{x}_{2}, \mathrm{x}_{3}\right)$ that are among the 26 neighbours of $\mathbf{x}$, defining a triangle ${ }^{1}$ that we denote $\overline{\mathbf{x}_{1} \mathbf{x}_{2} \mathbf{x}_{3}}$. There are 48 such triangles around $\mathrm{x}$ for the 26 connexity. To make the update procedure faster, we propose to consider only the simplexes $^{2}$ defined by a t-uple of three points of the 6-neighbors of $\mathbf{x}$. There are 8 such triangles (see Fig. 2), and by making this modification, the precision of the algorithm is lower but the algorithm is six times faster. To estimate $\mathcal{U}\left(\mathrm{x}_{\mathrm{m}}\right)$, in rou-

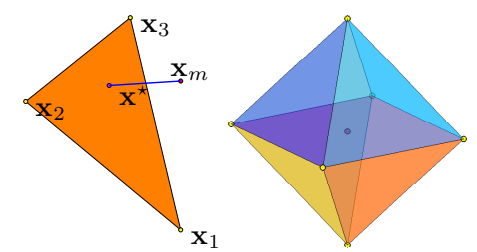

Figure 2. On the left Position of the optimal point on a simplex such as to minimize the geodesic distance to $\mathbf{x}$. On the right the considered simplexes.

tine UpdateScheme of Table 1, we say that the geodesic passing by $\mathbf{x}_{\mathrm{m}}$ comes from a triangle $\overline{\mathbf{x}_{1} \mathbf{x}_{2} \mathbf{x}_{3}}$ and then the time of arrival is given by:

$$
\mathcal{U}\left(\mathbf{x}_{\mathrm{m}}\right)=\min _{\mathbf{x} \in \mathbf{x}_{1} \mathbf{x}_{2} \mathbf{x}_{3}}\left\{\mathcal{U}(\mathbf{x})+\int_{\mathbf{x}}^{\mathbf{x}_{\mathrm{m}}} P\left(\gamma, \gamma^{\prime}\right)\right\}
$$

The term one wants to minimize is approximated by :

$$
f(\alpha)=\sum_{i=1}^{3} \alpha_{i} \mathcal{U}\left(\mathbf{x}_{i}\right)+\left\|\mathbf{x}-\sum_{i=1}^{3} \alpha_{i} \mathbf{x}_{i}\right\|_{\mathcal{M}(\mathbf{x})},
$$

where $\alpha=\left(\alpha_{1}, \alpha_{2}, \alpha_{3}\right)$, with $\sum_{i=1}^{3} \alpha_{i}=1$ since the point $\mathrm{x}$ is in the triangle (see figure 2 ). This equation follows Tsitsiklis's approximation [18]. The first term approximates the value of the minimal action map at the point $\mathbf{x}=\sum_{i=1}^{3} \alpha_{i} \mathbf{x}_{i}$ by a simple linear interpolation. And the second term approximates the remaining distance by considering the metric constant along the segment $\left[\mathbf{x}, \mathbf{x}_{\mathrm{m}}\right]$ equal to its value at point $\mathbf{x}_{\mathrm{m}}$. The function $f$ is convex and the constraints on $\alpha$, i.e $\sum_{i=1}^{3} \alpha_{i}=1$ and $\alpha_{i} \geq 0$, define a convex subset. Thus the minimization of $f$ can be done using classical optimization tools. See [7] for more details.

For each of the eight triangles, we get a value $u$. Finally, we choose the triangle giving the smallest value of $u$. This

\footnotetext{
${ }^{1}$ Such that $\mathbf{x}_{1}$ is a 6-connectivity neighbor, $\mathbf{x}_{2}$ is a 18-connectivty neighbor on the same face as $\mathbf{x}_{1}$, and $\mathbf{x}_{3}$ is a 26-connectivity neighbor on the same edge as $\mathbf{x}_{2}$.

${ }^{2}$ This time, all $\mathbf{x}_{i}$ are 6-connectivity neighbors, but on a same face of the cube surrounding $\mathbf{x}$, see figure 2 .
}

is the estimate returned by routine UpdateScheme in the Fast Marching algorithm described in table 1. Note that in order to approximate $\nabla \mathcal{U}$, computing the derivatives of $\mathcal{U}$ in the triangle using the estimate $\mathcal{U}\left(\mathrm{x}_{\mathrm{m}}\right)$ gives a consistent approximation of $\nabla \mathcal{U}\left(\mathrm{x}_{\mathrm{m}}\right)$ by the following:

$$
\nabla \mathcal{U}\left(\mathbf{x}_{\mathrm{m}}\right)=\left(\mathcal{U}\left(\mathbf{x}_{\mathrm{m}}\right)-\mathcal{U}\left(\mathbf{x}^{\star}\right)\right) \frac{\mathbf{x}_{\mathrm{m}}-\mathbf{x}^{\star}}{\left\|\mathbf{x}_{\mathrm{m}}-\mathbf{x}^{\star}\right\|},
$$

where $\mathbf{x}^{\star}$ is the minimizer of function $f$, see figure 2 left, and $\|$.$\| is the Euclidean norm. The computation of the gra-$ dient is very useful since it is used to solve the gradient descent described by equation (4).

\section{Optimally Oriented Flux : an Anisotropy Descriptor}

We are interested in the construction of a metric that extracts from the image the geometric information leading to reconstruction of vessels. This means that we wish to find an estimate for the local orientation and scale and a criterion on the local geometry to distinguish the presence of vessels from the background.

At the position $\mathrm{x}$ on a $2 \mathrm{D}$ image $I$, the amount of the image gradient projected along the axis $\mathbf{v}$ flowing out from a 2D circle $\partial D_{r}$ is measured as in [9],

$$
f(\mathbf{x}, \mathbf{v} ; r)=\int_{\partial D_{r}}((\nabla(G * I(\mathbf{x}+\mathbf{h})) \cdot \mathbf{v}) \mathbf{v}) \cdot \frac{\mathbf{h}}{|\mathbf{h}|} d a,
$$

where $G$ is a Gaussian function with a scale factor of 1 pixel, $r$ is the circle radius, $\mathbf{h}$ is the position vector along $\partial D_{r}$ and $d a$ is the infinitesimal length on $\partial D_{r}$. To detect vessels having higher intensity than the background region, one would be interested in finding the vessel direction which minimizes $f(\mathbf{x}, \mathbf{v} ; r)$, i.e. we are looking for:

$$
\arg \min _{\mathbf{v}} f(\mathbf{x}, \mathbf{v} ; r) .
$$

Using the divergence theorem, it can be shown that $f(\mathbf{x}, \mathbf{v} ; r)$ can be calculated using a simple convolution [9],

$$
f(\mathbf{x}, \mathbf{v} ; r)=\mathbf{v}^{T}\left\{\left(\partial_{i, j} G\right) * I * \mathbb{1}_{D_{r}}\right\} \mathbf{v},
$$

where $\left(\partial_{i, j} G\right)$ is the Hessian matrix of function $G$ and $\mathbb{1}_{D_{r}}$ is the indicator function of the disc $D_{r}$. By differentiating the above equation with respect to $\mathbf{v}$, minimization of function $f$ is in turn acquired as solving a generalized eigenvalue decomposition problem.

Solving the aforementioned generalized eigen decomposition problem gives two eigenvalues, $\lambda_{1}(\mathbf{x} ; r)$ and $\lambda_{2}(\mathbf{x} ; r)$ where $\lambda_{1}(\cdot) \leq \lambda_{2}(\cdot)$ and two eigenvectors $\mathbf{v}_{1}(\mathbf{x} ; r)$ and $\mathbf{v}_{2}(\mathbf{x} ; r)$, i.e. $\lambda_{1}(\mathbf{x} ; r)=f\left(\mathbf{x}, \mathbf{v}_{1}(\mathbf{x} ; r) ; r\right)$ and $\lambda_{2}(\mathbf{x} ; r)=$ $f\left(\mathbf{x}, \mathbf{v}_{2}(\mathbf{x} ; r) ; r\right) . \quad \mathbf{v}_{1}$ is perpendicular to the vessel direction and $\mathbf{v}_{2}$ is parallel to it when the chosen point $\mathbf{x}$ is on 


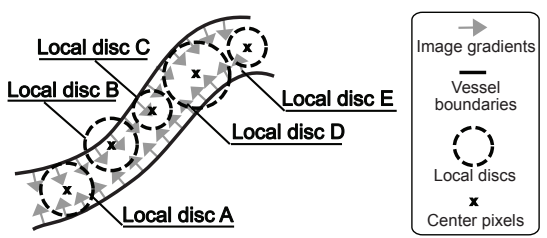

Figure 3. Five examples of computing OOF using different radii at various positions inside a vessel. Local discs A and B have the exact radius of the vessel. Local discs $\mathrm{C}$ and $\mathrm{E}$ have an undersized radius and Local disc $\mathrm{D}$ has an oversized radius.

the centerline and the scale $r$ is equal to the vessel radius. Along the vessel direction, $\mathbf{v}_{2}(\cdot)$, it is obvious that the magnitude of the eigenvalue $\lambda_{2}(\cdot)$ is small inside the vessel as image gradient magnitudes are minor along the vessel direction. Meanwhile, along the first eigenvector $\mathbf{v}_{1}(\cdot)$, the magnitudes of $\lambda_{1}(\cdot)$ varies according to the radius of the local disc and also the positions where OOF is evaluated. To illustrate this idea, five examples are presented in Figure 3.

For Local disc A, OOF is computed at the center of the vessel with its exact radius. As the evaluation of equation (7) is grounded on analyzing image gradients along the boundary of the disc, the OOF detection results are only induced when the disc boundary touches the object boundary. In equation (7), when $r$ is the vessel radius and the image gradients are projected along $\mathbf{v}_{1}$, the projected image gradients at the contacting positions between the boundary of Local disc A and the vessel boundaries are aligned along the orientation of the disc outward normal $\frac{\mathbf{h}}{|\mathbf{h}|}$. As such, the contacting positions between the disc boundary and the boundaries at the both side of the vessel contribute to the computation of equation (7). Consequently, a negative eigenvalue $\lambda_{1}(\cdot)$ with a large magnitude can be obtained.

It is worth mentioning that the magnitudes of $\lambda_{1}(\cdot)$ for Local discs B, C, D and E are smaller than that of Local disc A. For Local disc B, at the contacting positions between the disc boundary and the vessel boundary, the image gradients that point to the center line of the vessels are not aligned along the disc outward normal. It therefore suppresses the resultant magnitude of the dot product between the projected image gradient and the disc outward normal in equation (7).

On the other hand, for Local disc C, the disc boundary and the vessel boundary have only one contacting position that restraints the magnitude of $\lambda_{1}(\cdot)$. It is in contrast to the case of Local disc A where the disc touches the vessel boundaries on the both sides of the vessel. The Local disc D also returns a small magnitude eigenvalue $\lambda_{1}(\cdot)$ with the reasons similar to those of Local discs $\mathrm{B}$ and $\mathrm{C}$. The magnitude of $\lambda_{1}(\cdot)$ for Local disc $\mathrm{E}$ is insignificant and random as there is no overlapping between Local disc $\mathrm{E}$ and the vessel boundaries. Considering the small magnitudes of the first

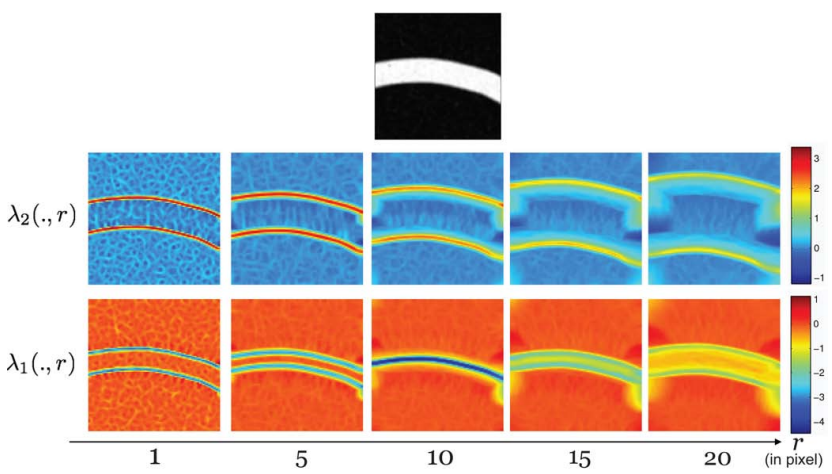

Figure 4. The OOF's eigenvalues computed on a 2D tubular structure. Top row: The synthetic image consists of a 10 pixels radius tube, where the intensity inside the structure is 255 and 0 for the background. Additive Gaussian noise with standard deviation 10 is added. The second and the third row: The second and the first eigenvalues extracted by the OOF method using different values of $r$ (radii).

eigenvalues in the cases of Local discs B, C, D and E, the first eigenvalue of OOF can attain the maximum negative value on Local disc A, where OOF is evaluated at the center of the vessel with its exact radius. Figure 4 shows the OOF's eigenvalues computed on a synthetic tube. In which, strong detection responses are acquired along the center line of the tube when OOF is evaluated with the same radius as the vessel radius (see the sub-image of $r=10$ in the bottom row of Figure 4). At four different positions of the synthetic tube (see figure 5a), the computation result of equation (7) using various values of $r$ and different projection axes $\mathbf{v}$ are shown in figures $5 \mathrm{c}$-f. As $\lambda_{2}(\mathbf{x} ; r)=f\left(\mathbf{x}, \mathbf{v}_{2} ; r\right)$, these figures clearly demonstrate second eigenvalue of OOF only attains its negative maximal at the center of the vessel with its exact radius.

To handle the vessels having various radii, a multi-scale approach should be used along with the OOF method. In [9], Law and Chung have proposed to normalize the OOF's eigenvalues by the sphere surface area when the OOF method is incorporated in a multi-scale approach for $3 \mathrm{D}$ image volumes. In the $2 \mathrm{D}$ case the eigenvalues are normalized by the circle perimeter $2 \pi r$.

It is important to point out that the flux introduced by Law and Chung in [9] is completely different than the fluxbased approach introduced by Vasilevskiy and Sidiqqi in [19] which is included in a global energy to minimize along the shape to find (for tubular structures segmentation), while our flux is a local feature which allows to define a local metric in order to minimize a path energy.

\section{How to construct the metric?}

$\mathrm{Li}$ and Yezzi [10] proposed a new variant of the classical, purely spatial, minimal path technique by incorporating an 


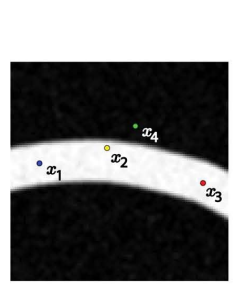

(a)

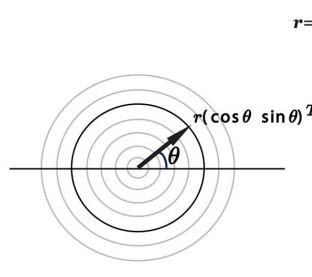

(b)

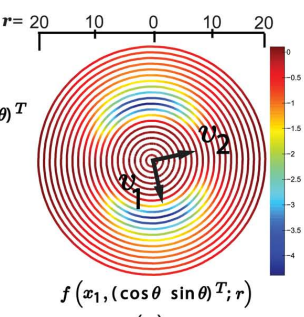

(c)

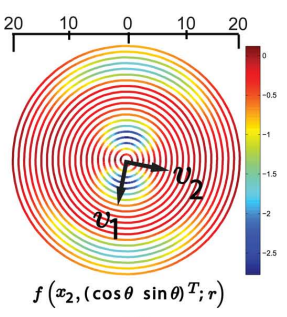

(d)

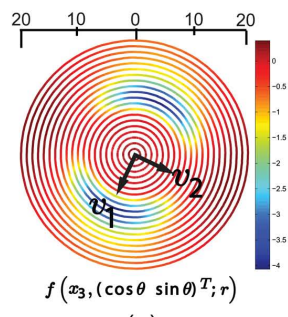

(e)

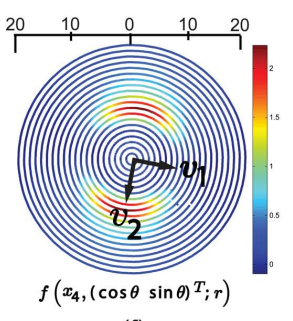

(f)

Figure 5. The plots of the values of $f(\mathbf{x}, \mathbf{v} ; r)$ as a function of projection axis $\mathbf{v}$ and radius $r$, obtained from the synthetic image shown in Figure 4 , at four different positions with various radii and projection axes. (a) Four interest positions, denoted as $\mathbf{x}_{1}, \mathbf{x}_{2}, \mathbf{x}_{3}$ and $\mathbf{x}_{4}$ are shown along with the original synthetic image presented in Figure 4. (b) An illustration regarding the polar coordinate system used for direction $\mathbf{v}$ in (c)-(f). (c)-(f) The plots of the values of $f(\cdot)$ and the corresponding eigenvectors, computed at the four different positions shown in (a), using various values of $r$ and different projection axes $(\cos \theta \sin \theta)^{T}$.

extra non-spatial dimension into the search space. The crucial step of this method is to build an adequate metric that drives the propagation. Li and Yezzi [10] proposed different isotropic potentials. The main drawback, as they mention, is that these potentials are very parameter dependent and they do not exploit the vessel orientation. Our main contribution is to improve Li and Yezzi method by adding to it an anisotropic formulation, and the anisotropic metric is constructed by extension of the OOF descriptor presented by Law et al. [9].

The 3D minimal path is found by minimizing the following energy:

$$
\int_{\gamma}\left\{\sqrt{\gamma^{\prime}(s)^{T} \mathcal{M}(\gamma(s)) \gamma^{\prime}(s)}\right\} \mathrm{d} s,
$$

where $\mathcal{M}$ is the anisotropic metric we want to construct. It is not natural to consider orientations on the $3^{\text {rd }}$ dimension, i.e. the radii dimension. Thus one can decompose by block the metric $\mathcal{M}$ as follows:

$$
\mathcal{M}(\mathbf{x}, r)=\left(\begin{array}{cc}
\tilde{\mathcal{M}}(\mathbf{x}, r) & 0 \\
0 & \mathcal{P}_{\text {radius }}(\mathbf{x}, r)
\end{array}\right)
$$

where $\tilde{\mathcal{M}}(\mathbf{x}, r)$ is a $2 \times 2$ symmetric definite positive matrix giving the spatial anisotropy and $\mathcal{P}_{\text {radius }}(\mathrm{x}, r)$ is the radius potential (also strictly positive).

Since the result given by the anisotropic minimal path method is very dependent on the metric, results inherit advantages and drawbacks of the constructed metric, thus we should be very careful with its construction. First, let us fix conditions on the desired metric. The spatial metric $\tilde{\mathcal{M}}$ has to be well oriented along the vessel centerline. And the radius potential $\mathcal{P}_{\text {radius }}$ has to be small for the adequate scale for any point of the image. $\sqrt{\mathcal{P}_{\text {radius }}}$ corresponds to the inverse speed for the radii dimension. Since $\tilde{\mathcal{M}}$ is symmetric definite positive, we can decompose it as follows: $\tilde{\mathcal{M}}()=.\sum_{i=1}^{2} m_{i}(.) \mathbf{u}_{i}(.) \mathbf{u}_{i}(.)^{T}$, where $0<m_{1} \leq m_{2}$ are the eigenvalues and $\mathbf{u}_{i}$ are the associated eigenvectors. The velocity of the propagating front along direction $\mathbf{u}_{i}$ is equal to $1 / \sqrt{m_{i}}$, for $i=1,2$.
As presented in section 3, for a point on the center line of the vessel, and when $r$ is equal to the exact vessel scale, the eigenvector $\mathbf{v}_{2}$ is parallel to the vessel, see figure 5. Thus the associated speed should be higher than the speed along the perpendicular direction $\mathbf{v}_{1}$. But the obtained eigenvalues satisfy $\lambda_{1} \leq \lambda_{2}$ and they may be negative. Since the metric $\tilde{\mathcal{M}}$ should be positive, we took:

$$
\begin{array}{r}
\tilde{\mathcal{M}}(.)=e^{\alpha \lambda_{2}(.)} \mathbf{v}_{1}(.) \mathbf{v}_{1}(.)^{T}+e^{\alpha \lambda_{1}(.)} \mathbf{v}_{2}(.) \mathbf{v}_{2}(.)^{T} \\
\mathcal{P}_{\text {radius }}(.)=\beta \exp \left(\alpha \frac{\lambda_{1}(.)+\lambda_{2}(.)}{2}\right)
\end{array}
$$

The constant $\alpha$ is controlled by an intuitive parameter, which is the maximal spatial anisotropy ratio: $\mu=$ $\max _{\mathbf{x}, r}\left\{\frac{\exp \left(\alpha \lambda_{2}(\mathbf{x}, r)\right)}{\exp \left(\alpha \lambda_{1}(\mathbf{x}, r)\right)}\right\}$. By choosing the maximal spatial anisotropy ratio $\mu$, the constant $\alpha$ is fixed. And by doing so, the anisotropy descriptor $\mathcal{M}$ becomes contrast invariant because the OOF is linear on the image. The parameter $\beta$ controls the radius speed. If $\mathcal{P}_{\text {radius }} \leq \exp \left(\alpha \lambda_{1}\right)$ then the Fast Marching propagation is faster for the radii than the spatial dimensions. If $\mathcal{P}_{\text {radius }} \geq \exp \left(\alpha \lambda_{2}\right)$ then the propagation is slower. One can tune parameter $\beta$ depending on the tubular structure one wants to extract. If its radius changes a lot then $\beta$ should be chosen such that the propagation on the radius dimension is faster. If not, $\beta$ is chosen such that the propagation is less sensitive on the radius dimension.

On figure 6 the constructed metric of figure 4 at some different scales is shown. Since we chose the same color range for the visualization, we can see that the directions are well detected, and that the optimal values are obtained along the centerline of the tube when the scale is equal to the tube radius. For our experiments, we took $\mu=10$ and $\beta$ such that $\max \left(\frac{e^{\alpha \lambda_{1}}}{\mathcal{P}_{\text {radius }}}\right)=5$, this means that in the worst case, the speed along the radii dimension is $\sqrt{5}$ times faster than the spatial dimensions. We did so, because we wanted our algorithm to be sensitive to the radii dimension. 


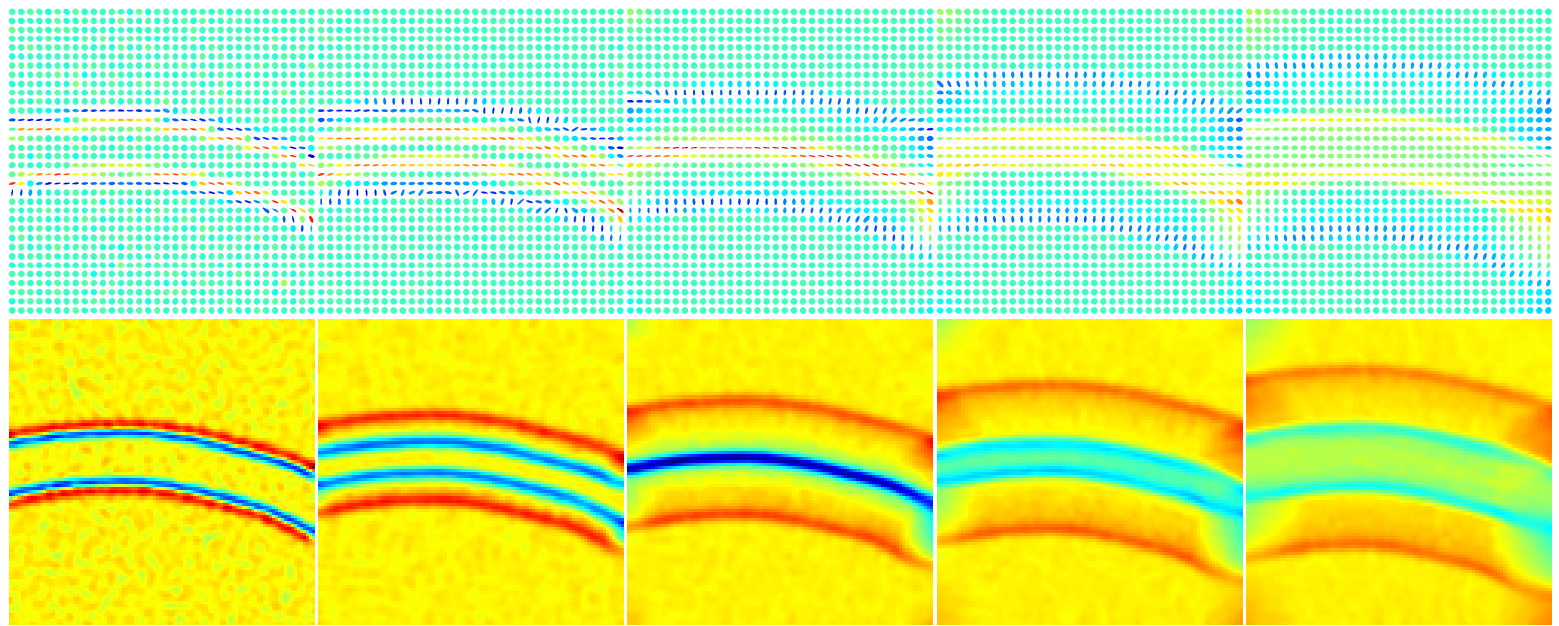

Figure 6. The constructed metric for different scales $r=1,5,10,15,20$ from left to right. The original image is shown in figure 5 (a), the radius of the structure is equal to 10 . We used the same color range for all images, so one can see that the optimal anisotropy is obtained along the centerline of the tubular structure when the scale $r$ is equal to the exact radius of the tube. On the top, we show a display of $\tilde{\mathcal{M}}(\mathbf{x}, r)^{-1}$ using small ellipses of eigenvalues. On the bottom, responses of $\mathcal{P}_{\text {radius }}$ are shown.
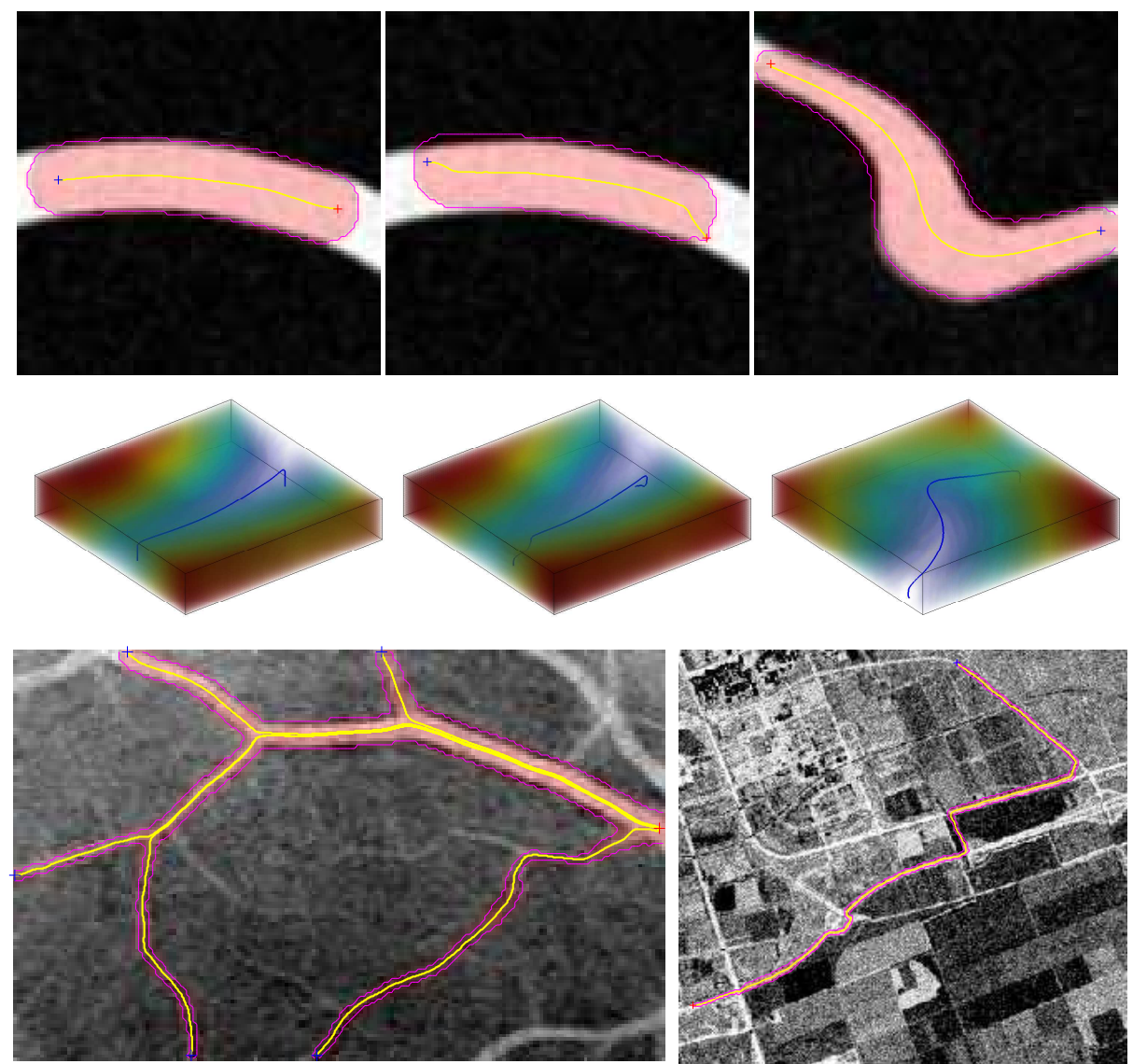

Figure 7. The red cross points are source points given by the user, and the blue ones are end points. On each case the segmented centerlines are displayed as well as the envelope of the moving discs. We show results on synthetic, medical and aerial images. In the middle, the associated minimal action map $\mathcal{U}$ as well as the $3 \mathrm{D}$ minimal path between the two selected points are shown (transparent visualization). 


\section{Experimental Results}

Our method is minimally interactive. First, the user has to precise if the desired vessels are darker or brighter than the background. So, we can consider different criteria on the signs of the eigenvalues. Then the scale range $\left[r_{\min }, r_{\max }\right]$, which corresponds to the range of radii of the vessel one wants to extract, is given by the user. Finally few points are required as source points or end points of the Fast Marching algorithm. We used the metric described in the previous section to find the minimal anisotropic path (as described in section 2) between two or more selected points (see figure 7). For any selected point, the associated radius is equal to the minimal radius $r_{\min }$ given by the user.

On figure 7, segmentation results on synthetic and real noisy 2D images are shown. On the first synthetic image, the source point and destination are selected on the centerline. The obtained tube is perfectly detected as well as the centerline. On the second image, the initial points are not centered. But the centerline given by our algorithm goes back fast to the real centerline. This makes our algorithm robust to initialization. The third synthetic image shows that our approach is robust to scale changing. On the last line of figure 7, segmentation results are shown on real noisy images. Obviously, these early experiments are not sufficient, and we are working on a quantitative comparison of our approach with existing ones like Siddiqi's [19] and others.

\section{Conclusion}

In this paper we have proposed a new general method for tubular structure extraction in 2D images. Our method exploit the orientation of the vessels by using the optimally oriented flux to construct a multi-resolution anisotropic metric that extracts from the image the local geometry and describes the vessels orientation and scales. Combining this metric with anisotropic minimal path technique, we were able to find a complete description of the tubular structure, i.e the centerline as well as the boundary. To summarize, our method is minimally interactive, robust to initialization, scale variations and bifurcations. In the future, we will extend our method to 3D vessels, and we will also work on the medical validation on coronary arteries.

\section{Acknowledgements}

We would like to thank Professor Anthony J. Yezzi for interesting discussions. This work was partially supported by ANR grant SURF -NT05-2_45825, and from the K S Lo Foundation and RGC612308.

\section{References}

[1] D. L. Chopp. Replacing iterative algorithms with single-pass algorithms. Proceedings of the National Academy of Sci- ences of the United States of America, 98(20):10992-10993, 2001.

[2] L. D. Cohen and R. Kimmel. Global minimum for active contour models: a minimal path approach. International Journal of Computer Vision, 24:57-78, 1997.

[3] T. Deschamps and L. Cohen. Fast extraction of minimal paths in 3D images and applications to virtual endoscopy. Medical Image Analysis, 5(4), Dec. 2001.

[4] E. W. Dijkstra. A note on two problems in connection with graphs. Numerische Mathematic, 1:269-271, 1959.

[5] J. Duncan and N. Ayache. Medical image analysis: Progress over two decades and the challenges ahead. PAMI, 22(1):85106, January 2000.

[6] R. F. Frangi, W. J. Niessen, K. L. Vincken, and M. A. Viergever. Multiscale vessel enhancement filtering. pages 130-137. Springer-Verlag, 1998.

[7] S. Jbabdi, P. Bellec, R. Toro, J. Daunizeau, M. PélégriniIssac, and $\mathrm{H}$. Benali. Accurate anisotropic fast marching for diffusion-based geodesic tractography. Journal of Biomedical Imaging, 2008(1):1-12, 2008.

[8] K. Krissian. Flux-based anisotropic diffusion applied to enhancement of 3-D angiogram. TMI, 21(11):1440-1442, 2002.

[9] M. W. K. Law and A. C. S. Chung. Three dimensional curvilinear structure detection using optimally oriented flux. ECCV, 4:368-382, 2008.

[10] H. Li and A. Yezzi. Vessels as 4D curves: Global minimal 4D paths to extract 3-D tubular surfaces and centerlines. IEEE Transactions on Medical Imaging, 26(9):1213-1223, 2007.

[11] Q. Lin. Enhancement, extraction, and visualization of $3 D$ volume data. $\mathrm{PhD}$ thesis, Linkopings Universitet, 2003.

[12] J. Melonakos, E. Pichon, S. Angenent, and A. Tannenbaum. Finsler active contours. IEEE Trans Pattern Anal Mach Intell, 30(3):412-423, 2008.

[13] D. L. Pham, C. Xu, and J. L. Prince. Current methods in medical image segmentation. Annual Review of Biomedical Engineering, 2(1):315-337, 2000.

[14] W. N. R. Manniesing, M.A. Viergever. Vessel enhancing diffusion a scale space representation of vessel structures. $\mathrm{Me}$ dIA, 10(6):815-825, 2006.

[15] Y. Sato, S. Nakajima, N. Shiraga, H. Atsumi1, S. Yoshida, T. Koller, G. Gerig, and R. Kikinis. Three-dimensional multi-scale line filter for segmentation and visualization of curvilinear structures in medical images. MedIA, 2(2):143168, 1998.

[16] J. A. Sethian. A fast marching level set for monotonically advancing fronts. Proceedings of the National Academy of Sciences, 93:1591-1595, 1996.

[17] J. A. Sethian and A. Vladimirsky. Fast methods for the eikonal and related hamilton- jacobi equations on unstructured meshes. Proceedings of the National Academy of Sciences, 97(11):5699-5703, May 2000.

[18] J. N. Tsitsiklis. Efficient algorithms for globally optimal trajectories. IEEE Transactions on Automatic Control, 40:1528-1538, 1995.

[19] E. Vasilevskiy and K. Siddiqi. Flux maximizing geometric flows. IEEE TPAMI, 24:1565-1578, 2002. 